Cronfa - Swansea University Open Access Repository

This is an author produced version of a paper published in :

Psychopharmacology

Cronfa URL for this paper:

http://cronfa.swan.ac.uk/Record/cronfa26237

\title{
Paper:
}

Kemp, A., Outhred, T., Saunders, S., Brunoni, A., Nathan, P. \& Malhi, G. (2014). Impact of escitalopram on vagally mediated cardiovascular function in healthy participants: implications for understanding differential age-related, treatment emergent effects. Psychopharmacology, 231(11), 2281-2290.

http://dx.doi.org/10.1007/s00213-013-3374-4

This article is brought to you by Swansea University. Any person downloading material is agreeing to abide by the terms of the repository licence. Authors are personally responsible for adhering to publisher restrictions or conditions. When uploading content they are required to comply with their publisher agreement and the SHERPA RoMEO database to judge whether or not it is copyright safe to add this version of the paper to this repository. http://www.swansea.ac.uk/iss/researchsupport/cronfa-support/ 
Post-print of: Kemp, A. H., Brunoni, A. R., Outhred, T., Saunders, S., Nathan, P. J., \& Malhi, G. S. (2013). Impact of escitalopram on vagally mediated cardiovascular function in healthy participants: implications for understanding differential age-related, treatment emergent effects.

Psychopharmacology. doi:10.1007/s00213-013-3374-4

The published version of this article is available here: http://dx.doi.org/10.1007/s00213-013-3374-4

* Corresponding author: Andrew H. Kemp, Email: andrew.kemp@sydney.edu.au or andrew.kemp@hu.usp.br; Telephone: +55 11 3091-9300; Fax: +55 11 3815-9631 


\section{Impact of Escitalopram on Vagally Mediated Cardiovascular Function in Healthy Participants: Implications for Understanding Differential Age-Related, Treatment-Emergent Effects}

Andrew H. Kemp ${ }^{1,2,3,4^{*}}$, Tim Outhred ${ }^{1,2}$, Sasha Saunders ${ }^{1}$, Andre R. Brunoni ${ }^{4}$, Pradeep J. Nathan ${ }^{5,6}$, Gin S. Malhi ${ }^{2,3}$

${ }^{1}$ SCAN Research and Teaching Unit, School of Psychology, University of Sydney NSW 2006, Australia

2 Discipline of Psychiatry, Sydney Medical School, University of Sydney, Royal North Shore Hospital NSW 2065, Australia

${ }^{3}$ CADE Clinic, Department of Psychiatry, Royal North Shore Hospital NSW 2065, Australia

${ }^{4}$ University Hospital, University of São Paulo, São Paulo, Brazil

${ }^{5}$ Department of Psychiatry, University of Cambridge, Cambridge CB2 1QB, United Kingdom

${ }^{6}$ School of Psychology and Psychiatry, Monash University, Victoria, Australia

\section{Acknowledgements}

The authors would like to acknowledge the support of the following people throughout the project: Kristi Griffiths, Sasha Saunders, Kristy-Lee Feilds, Mimi Leith, Marijke Braeken, Julie Ji, Daniel Quintana, James Heathers, Caroline Fields, Jonathan Kreiger, Sara Shahrestani, Camilla Hanson, and Matthew Beauregard. 


\section{Funding and Disclosure}

This research was supported by an Australian Research Council Discovery Project Grant (DP0987332), a National Health and Medical Research Council (NHMRC) Project Grant (464863) and a NHMRC Career Development Award (571101) awarded to AHK. The authors AHK and TO are currently supported by an International Research Professorship from the Universidade de São Paulo and an Australian Postgraduate Award, respectively. PJN is an employee at GlaxoSmithKline Pharmaceuticals and holds shares in the company. GSM has received research support from AstraZeneca, Eli Lilly, Organon, Pfizer, Servier, and Wyeth. He has been a speaker for AstraZeneca, Eli Lilly, Janssen Cilag, Lundbeck, Pfizer, Ranbaxy, Servier, and Wyeth. He has been a consultant for AstraZeneca, Eli Lilly, Janssen Cilag, Lundbeck, and Servier. SS and ARB declare no potential conflict of interest. 


\begin{abstract}
Rationale: Black box warnings for young adults under the age of 25 years indicate that antidepressants may increase risk of suicide. While underlying mechanisms for age-related treatment effects remain unclear, vagally mediated cardiovascular function may play a key role. Decreased heart rate (HR) and an increase in its variability (HRV) improve ones capacity to adapt to environmental stress and attenuate risk for suicide. Objectives: Using a double blind, randomized, placebo-controlled, crossover, mixed experimental study, we examine whether a single-dose of escitalopram (20mg) attenuates cardiovascular responses to stress under experimental conditions, and determine whether age moderates these effects. Methods: Forty-four healthy females received a single dose of escitalopram $(20 \mathrm{mg})$ and placebo treatment separated by a one-week interval (> 5 half-lives). HR and high frequency HRV (HF HRV n.u.; 0.15-0.40 Hz) were measured during resting state and stress. Results: While escitalopram attenuated the increase in HR and increased HF HRV, these moderate to large effects were only significant in participants over 25 years of age. No beneficial cardiovascular effects of escitalopram were observed in those under the age of 25. Conclusions: Maturational differences in development of the prefrontal cortex - a critical region in the central network of autonomic control - may underpin these differential findings. This study provides a theoretical framework on which future research on treatment-emergent suicidality in clinical populations could be based.
\end{abstract}

Keywords: heart rate, heart rate variability, escitalopram, SSRI, antidepressant, stress, age, young adults, maturation 


\section{Introduction}

There is a growing body of evidence to suggest that adolescents and young people differentially respond to antidepressant treatment compared to more mature adults within the acute depressive episode (Barbui et al. 2009; Gibbons et al. 2012; Hammad et al. 2006a; Stone et al. 2009a). While underlying mechanisms for the differential treatment effects of age remain unclear (including an increase in suicidality), vagally mediated cardiac function may play a key role. A large body of literature suggests that the cardiovascular system may play a role in mental and physical health (see Kemp and Quintana 2013 for review). Most relevant for the current study is the link to psychological flexibility (Kashdan and Rottenberg 2010), the role of the vagal nerve in the inhibition of sympathetic nervous system responses to environmental stress (Thayer et al. 2009) and epidemiological findings reporting an association between vagally mediated cardiovascular function and suicidality (Åberg et al. 2013; Lemogne et al. 2011). Here we examined whether age-dependent differences in responses to acute administration of escitalopram - a first-line treatment option for moderate to severe major depressive disorder in adolescents (Ahn and Patkar 2011) - may be underpinned by differential cardiovascular responses; this is the hypothesis of the present study. We conducted a double blind, randomized, placebo-controlled, crossover, mixed experimental study on a well-characterized sample of healthy participants to avoid confounds associated with study of clinical samples. Our study lays an important theoretical framework for better understanding differential age-related responses to the early effects of antidepressant medication.

Vagally mediated cardiovascular function - including heart rate (HR) and its variability (HRV) - reflects the function of the central autonomic network (CAN) including the PFC, cingulate, insula, amygdala and brainstem structures (Thayer et al. 2009). The inhibitory role of the PFC is critical for overriding automatic and prepotent responses associated with subcortical structures such as the amygdala and medullary cardioacceleratory circuits (Thayer et al. 2009). However, adolescence and young adulthood is a developmental period in which the prefrontal cortex is often unable to provide sufficient top-down control over subcortical structures (Casey et al. 2008), leading to impulsive behavior and, in clinical populations, suicidality, especially in the face of emotional stress. Recent epidemiological studies have highlighted a role for vagally mediated cardiovascular function in suicidal behaviours. One study on more than 320,000 participants reported that an increase in heart rate by 10 additional beats per minute increases the risk of completed suicide by $19 \%$ to $37 \%$ over a follow-up period of 9 years (Lemogne et al. 2011). A more recent study on more than 1,000,000 participants reported that reduced cardiovascular fitness increases risk of suicide attempt or death by suicide by $79 \%$ over a 42 year follow-up period ( $\AA$ berg 
et al. 2013). Other studies further highlight a role for vagally mediated cardiovascular function in stress resilience (Hanson et al. 2013; Oldehinkel et al. 2008).

The serotonin (5-HT) system has been associated with impulsivity (Depue and Spoont 1986; Robbins and Crockett 2009), aggression (Carrillo et al. 2009) and suicide (Mann 2003; Mann 1998), and is a major target for the treatment of depression. The 5-HT system also plays a key role in regulating and maintaining cardiovascular function (Côté et al. 2004). Critically, acute manipulation of the 5-HT system has demonstrable effects on the cardiovascular system and impulsivity (Booij et al. 2006; Kemp and Nathan 2004), providing a useful model to understand the mood disorders and early effects of treatment. While researchers (Straneva-Meuse 2004) have proposed that SSRI antidepressants exert their benefits through direct action on the biological substrates of the stress response - including a blunting of blood pressure, myocardial responses, and cortisol reactivity under stress - other research indicates that they may also have adverse cardiovascular effects, including reduced parasympathetic nervous system activity (Licht et al. 2010). The increased suicidality in adolescents and young adults during the early treatment phase (Barbui et al. 2009; Gibbons et al. 2012; Hammad et al. 2006a; Stone et al. 2009a) suggests that these individuals may not benefit from the anxiolytic effects of SSRI antidepressants. There is substantial remodeling and maturation of the neurotransmitter systems - including 5-HT - during adolescence and into adulthood that may impact on the beneficial effects of antidepressants (Crews et al. 2007; Davey et al. 2008). Experimental animal research has shown that adolescent rats treated with paroxetine do not display the expected antidepressant effects on the forced swim test (FST) (Karanges et al. 2011). Furthermore, this study reported that two of the paroxetine-treated adolescents died unexpectedly after the FST suggesting a compromised response to physical stress (Karanges et al. 2011) (see also Karanges and McGregor 2011a for review). In an extension of this earlier study, we found (Karanges et al. 2013) that paroxetine-treated adolescent rats display a profile of hippocampal protein expression implicated in apoptosis, oxidative stress and monoaminergic neurotransmission. These findings provide experimental support for the epidemiological studies reporting 'paradoxical' antidepressant responses to SSRI treatment in young persons.

Here we examine the impact of acute escitalopram administration on HR and HRV under resting state and stress, and determine whether age moderates this effect. We wanted to determine whether escitalopram adversely impacts on cardiovascular function in late adolescence and young adulthood (participants aged under 25 years) - a finding that would support previous research indicating adverse effects of antidepressants in youth (Barbui et al. 2009; Hammad et al. 2006a; Stone et al. 2009a). Alternatively, participants in late adolescence and young adulthood may simply 
not experience the beneficial anti-panic properties that have been reported for escitlaopram (Hogg et al. 2006), a finding that would instead support the conclusions drawn by Gibbons and colleagues (Gibbons et al. 2012). The following hypotheses were made: (1) HR and HRV will increase and decrease under stress, respectively (main effect of task); (2) these responses will be attenuated by acute escitalopram treatment (main effect of treatment); and, (3) these responses will be moderated by age (interaction effects with task and treatment). We did not expect escitalopram to have favorable effects on vagally mediated cardiovascular function in the younger age group.

\section{Methods}

\section{Subjects}

Forty-four female participants completed the experiment (Fig 1). Sample size represents a good balance of improved statistical power over prior studies - studies on healthy participants with between-subject experimental designs have generally reported on samples with less than 20 participants (e.g. (Booij et al. 2006; Pohl et al. 2003)) - and ethical considerations relating to administration of antidepressants to young healthy participants. All participants provided informed consent in accordance with National Health and Medical Research Council (NHMRC) guidelines. This study was registered with the Australian and New Zealand Clinical Trials Registry (ANZCTR; ACTRN12611000719932) and was conducted at the CADE Clinic, a research unit in the Discipline of Psychiatry at Royal North Shore Hospital, Australia. Recruitment began in July 2011 and concluded July 2012. No changes in eligibility criteria were applied throughout the study. Exclusion criteria were as follows: age $<18$ or $>50$ years, history of psychiatric illness or antidepressant use, mild to severe depression or anxiety symptoms assessed using the 9-item depression module Patient Health Questionnaire (PHQ-9) and the 7-item generalized anxiety scale (GAD-7) (Kroenke et al. 2001; Spitzer et al. 2006), use of medications which interact with escitalopram (minimum one month medication free was acceptable), alcohol use disorders or regular illicit drug use, serious medical conditions, neurological disorders, brain injury, loss of consciousness for more than five minutes, history of stroke, or pregnancy (tested at each session). Although important to investigate effects in participants younger than 18, we excluded these participants from the current study for ethical reasons.

\section{Experimental Design}

The present study employed a double blind, randomized, placebo-controlled, crossover, mixed experimental study with age as a between-subjects factor (under 25 years of age versus those 25 years of age and over), while treatment and task were within-subjects factors. The age of 25 was selected as a cut-off because it is consistent with: 1) issued US Food and Drug Administration 
(FDA) issued black box warnings on treatment-emergent suicidality in young adults under the age of 25,2) meta-analyses supporting the FDA's decision (Hammad et al. 2006b) and others since (Barbui et al. 2009; Stone et al. 2009b), and 3) neuroimaging evidence indicating that the PFC continues to mature up into early adulthood (Casey et al. 2008).

An independent statistician generated the randomization list using standard software, and a pharmacist dispensed treatment in labeled containers in accordance with this list. Everyone involved in the study (researchers, participants, and clinicians) were blinded to the treatment condition until all assessments were completed. Participants were randomized to treatment order, and administered either a saccharin placebo or $20 \mathrm{mg}$ of escitalopram on their first visit and the alternate treatment on their second visit. The pharmacokinetic profiles of escitalopram do not differ between adolescents and young adults indicating that dosage adjustment according to age is not required (Rao 2007). A one-week interval separated visits in order to ensure sufficient washout of at least 5 half-lives (Anonymous 2012; Sogaard 2005). Participants were administered twice the recommended starting dose (Anonymous 2012) to maximize receptor occupancy (Kasper et al. 2009).

HRV data was collected at the same time of day for both visits, to control for circadian changes in HR. Participants abstained from caffeine on the morning of the experiment to minimize cross-session changes in heart rate and blood pressure. Upon arrival at each session, participants completed pregnancy tests and provided saliva samples $(1 \mathrm{~mL})$ in order to test for progesterone and estradiol concentration (see supplemental information). After treatment administration, participants waited three hours before psychophysiological recordings began to allow escitalopram to reach expected peak blood plasma levels ( $\mathrm{t}_{\max }=3.0 \pm 1.5$ hours; (Sogaard 2005)). A standardized snack was provided one and a half hours after treatment administration in order to control for changes in autonomic balance arising as a result of hunger and food intake. In order to examine the potential impact of treatment unblinding at each session, participants reported whether they experienced side effects and guessed their treatment condition (see supplemental information).

HR and HF HRV (normalized units, n.u.) (0.15-0.40 Hz) recordings were made under two conditions: (1) a 5-minute resting state and (2) a 5-minute social stress task, using a Polar RS800CX heart rate monitor. These devices have excellent reliability and validity - in comparison to the electrocardiogram (ECG) - especially when data is collected from young, healthy individuals in a supine position and analysis is conducted on normalized values (Gamelin et al. 2006; Weippert et al. 2010) (see also Quintana et al. 2012), as was done for the current study. This device is wirelessly connected to an electrode strap, wetted with saline solution $(0.9 \% \mathrm{NaCl}$; to simulate sweat and ensure conductivity) and worn just beneath the chest. For the resting state recording, participants 
were instructed to breathe normally, remain still and silent, and keep their eyes open in a seated position. The serial subtraction mental arithmetic component of the Trier Social Stress Test (Kirschbaum et al. 1993) was used for the stress condition, since it has been shown to reliably increase biomarkers of stress (Kirschbaum et al. 1993). The experimenter was seated in close proximity to the participant and acted in a formal manner, in order to further increase stress and social pressure.

\section{Data Analysis}

All HR and HF HRV data was extracted and manually inspected on Polar ProTrainer 5 software prior to analysis. Data quality was excellent, consistent with recordings under stationary conditions. Data was analyzed using Kubios software (available at: http://kubios.uku.fi). After applying a medium-level artifact correction to all data files, the average HR (beats/minute; BPM) and HF HRV (normalized units, n.u.) $(0.15-0.40 \mathrm{~Hz})$ over each treatment and task condition was calculated. Two analyses of variance (ANOVAs) were performed including one for HR and another for HF HRV variables to determine the presence of a three-way interaction (Task, Treatment, Age Group). A factor of "treatment order" was not included in statistical analysis; order effects are rare, are generally underpowered even when an appreciable effect is present and sufficient drug washout ameliorates such effects (Mills et al. 2009; Senn 1994; Senn et al. 2004). Cohen's $d$ effect size statistics were calculated for each pairwise comparison. Cohen's guidelines (Cohen 1992; Cohen 1988) identify 0.2, 0.5, and 0.8 as small, medium and large effects, respectively. Eta-squared ( $\eta 2$ ) was reported for ANOVA effects as an indicator of effect size $($ small $=.01$ medium $=.06$, large $=$ .14; (Cohen 1992; Cohen 1988)).

\section{Results}

Participant Characteristics

No differences were observed between age groups on any of the following variables: ethnicity, BMI, hormonal contraceptive use, menstrual phase in either the placebo and drug conditions, experience of side effects, correct treatment condition guessing, alcohol consumption, smoking status, MDD or GAD symptoms, overall change in state anxiety as measured using the STAI, or time spent performing vigorous physical activity (Table 1). Years of education and time spent sedentary in the past week were significantly greater in the 25 years and over group relative to those under the age of 25. 
The impact of state anxiety, time spent sedentary and time spent performing vigorous activity - factors associated with current cardiac health and stress reactivity - on HR and HF HRV was examined. For HR, significant findings were observed for time spent sedentary $(F[1,35]=$ $\left.5.777, p=.022, \eta^{2}=.180\right)$ and the interaction between state anxiety and sedentary activity $(F[1,35]$ $\left.=4.533, p=.040, \eta^{2}=.115\right)$ were observed. These findings indicate that between-subjects factors associated with cardiac health and functioning explain some of the variance in HR. For HF HRV, a significant interaction between state anxiety and vigorous activity was observed, $F(1,35)=4.273, p$ $=.046, \eta^{2}=.109$, again indicating that some variance in the HF measure is explained by factors associated with current cardiac health and functioning. No significant effects of other betweensubjects factors - including the experience of side effects and correct treatment condition guessing - on HR or HF HRV variables were observed. State anxiety, time spent sedentary and performing vigorous activity were therefore entered as covariates in analyses and these results are reported below. (Non-covaried findings are reported in supplementary information). It is important to note that key findings were obtained with and without use of covariates.

\section{Within Subjects Factors}

\section{Main Effect of Task}

For HR, a significant main effect of task condition was observed, $F(1,35)=37.763, p<$ $.001, \eta^{2}=.519$. In comparison to the rest condition, follow-up analysis revealed that HR was significantly increased under stress, $t(35)=13.241, p<.001, d=4.476$. This main effect of task was also observed in the HF HRV results, $F(1,35)=11.496, p=.002, \eta^{2}=.247$. In comparison to the rest condition, follow-up analysis revealed that HF was significantly reduced under stress, $t(35)$ $=8.564, p<.001, d=2.895$. (Fig 2, row 1). In summary, HR increased and HRV decreased under stress, findings associated with a large effect size.

\section{Main Effect of Treatment}

For HR, a significant main effect of treatment condition was found, $F(1,35)=4.865, p=$ $.034, \eta^{2}=.122$. Follow-up analysis revealed that HR was significantly reduced under escitalopram in comparison to placebo, $t(35)=2.038, p<.001, d=1.598$. For HRV, a main effect of treatment condition was also observed, $F(1,35)=4.514, p=.041, \eta^{2}=.114$. Follow-up analysis revealed that HF was greater under escitalopram than placebo, $t(35)=2.038, p=.049, d=0.689$ (Fig 2, row 2). In summary, HR reduced and HRV increased with escitalopram treatment, findings associated with a moderate (HRV) to large (HR) effect size. 


\section{Treatment $\times$ Task Interaction}

For HR, escitalopram decreased HR in the rest $(t[35]=3.271, p=.002, d=1.106)$ and stress conditions $(t[35]=4.757, p<.001, d=1.608)($ Fig 2 , row 3, column 1$)$, however, a significant treatment $\times$ task interaction effect was not observed. For HF HRV, a significant interaction effect of treatment $\times$ task condition was observed, $F(1,35)=7.289, p=.011, \eta^{2}=.172$. Follow-up analyses revealed that HF was significantly greater under escitalopram during the rest condition, $t(35)=$ 2.230, $p=.032, d=0.754$. (Fig 2, row 3, column 2). In summary, treatment with escitalopram decreased HR in the rest and stress conditions, while HF HRV was increased during the rest condition only, reflecting the difficulty of increasing parasympathetic function during stress. All findings were associated with a large effect size.

\section{Interactions of Within Subjects and Between Subjects Factors ${ }^{\text {ii }}$}

\section{Treatment $\times$ Age Group Interaction}

No significant treatment $\times$ age group interaction effects were observed for either HR or HF HRV.

\section{Task $x$ Age Interaction}

No significant task $\times$ age group interaction effects were observed for either HR or HF HRV.

\section{Treatment $\times$ Task $\times$ Age Group Interaction}

A treatment $\times$ task $\times$ age group interaction for HR was observed at trend levels, $F(1,35)=$ $3.778, p=.060, \eta^{2}=.097$. Follow-up analyses revealed that, for those aged 25 years and over, HR during the rest condition was significantly decreased under escitalopram when compared with placebo, $t[35]=2.379, p=.021, d=0.804$ (Fig 3, row 1, column 1). A more pronounced effect was found during the stress condition, such that those aged 25 years and over displayed significantly decreased HR under escitalopram when compared with placebo $t[35]=4.471, p<.001, d=1.512$ (Fig 3, row 1, column 1). While these findings in those aged 35 years and over were associated with a large effect size, no significant effects were observed for those aged under 25 years (Fig 3, rows 1 and 2, column 1). Complementing our HR findings from ANOVA, regression analysis revealed more attenuation of stress by escitalopram with increasing age, $\left(p=0.045, r^{2}=0.092\right)$, a finding associated with a moderate effect size (Fig 4). For HF HRV, a significant interaction effect of treatment $\times$ task $\times$ age group was also observed, $F(1,35)=6.314, p=.017, \eta^{2}=.153$. In comparison to placebo, follow-up analysis revealed that HF HRV was greater under escitalopram 
during the rest condition for those only over the age of 25 years $(t[35]=2.890, p=.007, d=0.977$; see Fig 3, row 1, column 2). No such change was observed for those under the age of 25 years.

\section{Discussion}

Research on vagally mediated cardiovascular effects of antidepressants may have important implications for better understanding age-related treatment-emergent side effects. Confirming our hypotheses, we show here that age moderates acute treatment effects on HR and HRV - key measures of cardiovascular function under tonic inhibitory control of the vagus nerve. Key findings from the present study were that (1) HR was increased and HRV decreased under stress (main effect of task), providing an important validation of our stressor, 2) HR was decreased and HRV increased under escitalopram (main effect of drug), indicating that acute administration of SSRIs have beneficial inhibitory effects on cardiovascular function, 3) HR of participants aged 25 years and over was decreased by escitalopram and this effect was most pronounced during stress (treatment $\mathrm{x}$ task $\mathrm{x}$ age interaction), and 4) HF HRV was increased under escitalopram during rest only (treatment $\mathrm{x}$ task interaction), reflecting the difficulty of increasing parasympathetic function under stress. This latter effect was significant only in those aged 25 years and over (treatment $\mathrm{x}$ task $\mathrm{x}$ age interaction). Importantly, task effects are not dependent on age (no interaction between task and age was observed) highlighting that maturational differences relate to the capacity of treatment to impact on cardiovascular responses to stress, rather than differential age-related responses to task, subsequently impacting on treatment. These findings provide a potential psychophysiological explanation for recent findings (Gibbons et al. 2012) indicating that unlike adults 25 years of age and over, antidepressant treatment is not successful in ameliorating reductions in vagally mediated cardiovascular function in those aged under 25 years (Gibbons et al. 2012). As vagally mediated cardiovascular function plays an important role in psychological flexibility (Kashdan and Rottenberg 2010), stress resilience (Oldehinkel et al. 2008; Thayer et al. 2009) and even, suicidality (Åberg et al. 2013; Lemogne et al. 2011), our findings have important implications for the capacity of escitalopram to modulate responses to stress in a flexible and adaptive manner in adolescents and young adults.

We suggest that maturation of the PFC may underpin the differential attenuation of cardiovascular responses to stress following administration of escitalopram, findings reported here. While cortical changes do occur in childhood and adolescence - particularly in the parietal cortex (Cohen et al. 1992) - research indicates that frontal regions develop well into the 20's (23-30 years) relative to adolescents (12-16 year olds) (Sowell et al. 1999). Adolescence and young adulthood is characterized by PFC immaturity and insufficient top-down control of subcortical 
structures (Casey et al. 2008; Sowell et al. 1999). These changes may be underpinned by decreases in gray matter paralleled by increases in myelination (Benes et al. 1994; Yakovlev and Lecours 1967). Although the serotonin system develops to near maturity in the first years of life, maturational changes continue to occur throughout adolescence and into adulthood (Crews et al. 2007; Karanges and McGregor 2011b). The adolescent brain is characterized by increased serotonin levels and 5-HT1A and 5-HT2A receptor densities (Crews et al. 2007; Murrin et al. 2007), but lower 5-HTT density and serotonin turnover (Crews et al. 2007; Moll et al. 2000). The serotonin system is diverse and neurochemically complex, however, it is understood to play a critical inhibitory role in the PFC (Fuster 2008; Puig and Gulledge 2011) and is implicated in a variety of behaviours including impulsivity (Depue and Spoont 1986; Robbins and Crockett 2009), aggression (Carrillo et al. 2009) and suicide (Mann 2003; Mann 1998). Maturation of the PFC and the serotonergic system will therefore limit the beneficial effects of escitalopram on vagally mediated cardiovascular function. Our findings indicate that escitalopram does not improve the capacity for flexible and adaptive responding to stress in adolescents and young adults, unlike that for those aged over 25 years of age. The failure of escitalopram to increase cardiac vagal control in participants under the age of 25 suggests that ongoing neurochemical, functional and structural maturation in young adults may limit the beneficial effects of escitalopram. We suggest that antidepressants may have greater impact on vagally mediated cardiovascular function in those 25 years of age and older, because of their action on a more developed PFC - including 5-HT pathways - and downstream parasympathetic inhibition of cardiovascular responses to stress. Further studies are required to further understand the mechanisms underpinning paradoxical antidepressant responses in adolescents and young adults.

A limitation of the present study was potential unblinding of treatment - participants correctly guessed treatment condition above chance levels when they experienced side effects (see supplementary information), consistent with those typically experienced with escitalopram (Cipriani et al. 2009). However, guessing and side effects did not differentially affect HR or HF HRV (supplementary information), indicating that these factors are unlikely to have contributed and confounded the results of the present study. Nevertheless, future research may consider employing an active placebo condition (specifically, a treatment that replicates the side effects of the antidepressant of interest) in order to control for the potential impact of side effects on stress reactivity. Another possible limitation of the present study was that a female-only sample was employed, however differential impacts of hormonal or menstrual status on HR or HRV measures were not observed (see supplemental information). Female participants were selected for study as they are more responsive to SSRI treatment (Khan et al. 2005), have greater physiological 
responses to stress induction ( $\mathrm{Li}$ et al. 2009) and are twice as likely to be diagnosed with affective disorders (Altemus 2006). It is also important to acknowledge that findings reported here are based on acute administration of escitalopram, while epidemiological findings associated with suicidality in adolescents and young adults are associated with sub-chronic treatment (i.e. the early activating effects of antidepressant treatment). However, early psychophysiological changes may precede any overt, observable change in behavior (Kemp et al. 2004; Kemp and Nathan 2004), and these early changes provide a platform on which later changes in behavior are based (Harmer et al. 2009). Finally, while we focus on a healthy participant sample, we avoid the many confounds associated with studies on patients including non-randomized-controlled designs, difficulty in distinguishing between the effects of medication versus illness severity, cardiovascular health, sex, and hormonal status.

In conclusion, the present study found for the first time that acute administration of escitalopram does not attenuate cardiovascular responses to stress in those under 25 years of age, providing a potential psychophysiological mechanism for better understanding findings on agerelated treatment-emergent suicidality in epidemiological studies (Gibbons et al. 2012). Future epidemiological research on clinical populations should consider incorporating measures of cardiac vagal control to further examine whether age- and treatment-emergent suicidality are associated with a lack of cardiac vagal control.

Supplementary information is available at the Psychopharmacology website. 
Table 1

Participant demographics

\begin{tabular}{|c|c|c|c|}
\hline & $\begin{array}{l}\text { Under } 25 \mathrm{~s} \\
(n=26)\end{array}$ & $\begin{array}{c}25 \text { and over } \\
(n=18)\end{array}$ & $\begin{array}{c}\text { Group } \\
\text { Differences }\end{array}$ \\
\hline Age & 20.54 & 29.50 & $t(42)=6.66$ \\
\hline (years; SD) & $(2.01)$ & $(6.46)$ & $p<.001$ \\
\hline Education & 14.88 & 19.17 & $t(42)=7.51$ \\
\hline (years; SD) & $(1.66)$ & $(2.12)$ & $p<.001$ \\
\hline $\begin{array}{l}\text { Ethnicity } \\
(\mathrm{C} / \mathrm{NC})\end{array}$ & $2 / 24$ & $0 / 18$ & $\begin{array}{c}\chi^{2}(1)=1.451 \\
p=.228\end{array}$ \\
\hline BMI & 22.79 & 22.91 & $t(42)=0.13$ \\
\hline$\left(\mathrm{kg} / \mathrm{m}^{2} ; \mathrm{SD}\right)$ & $(3.46)$ & $(2.38)$ & $p=.896$ \\
\hline $\begin{array}{l}\text { Hormonal contraceptive use } \\
(\mathrm{Y} / \mathrm{N})\end{array}$ & $12 / 14$ & $9 / 9$ & $\begin{array}{c}\chi^{2}(1)=0.063 \\
p=.802\end{array}$ \\
\hline $\begin{array}{l}\text { Menstrual phase Placebo } \\
(\mathrm{F} / \mathrm{M} / \mathrm{L})\end{array}$ & $11 / 7 / 8$ & $5 / 7 / 6$ & $\begin{array}{c}\chi^{2}(2)=1.118 \\
p=.572\end{array}$ \\
\hline $\begin{array}{l}\text { Menstrual phase Drug } \\
(\mathrm{F} / \mathrm{M} / \mathrm{L})\end{array}$ & $11 / 7 / 8$ & $5 / 9 / 4$ & $\begin{array}{c}\chi^{2}(2)=2.460 \\
p=.292\end{array}$ \\
\hline $\begin{array}{l}\text { Side effects } \\
(\mathrm{Y} / \mathrm{N})\end{array}$ & $12 / 14$ & $9 / 9$ & $\begin{array}{c}\chi^{2}(1)=0.063 \\
p=.802\end{array}$ \\
\hline $\begin{array}{l}\text { Correct treatment guess } \\
(\mathrm{Y} / \mathrm{N})\end{array}$ & $7 / 19$ & $6 / 12$ & $\begin{array}{c}\chi^{2}(1)=0.210 \\
p=.647\end{array}$ \\
\hline AUDIT Total & 7.54 & 5.11 & $t(42)=1.69$ \\
\hline (SD) & $(5.57)$ & $(3.16)$ & $p=.103$ \\
\hline $\begin{array}{l}\text { Smoking } \\
(\mathrm{Y} / \mathrm{N})\end{array}$ & $0 / 26$ & $0 / 18$ & - \\
\hline PHQ-9 & 1.85 & 1.11 & $t(42)=1.89$ \\
\hline (SD) & $(1.43)$ & $(1.02)$ & $p=.069$ \\
\hline GAD-7 & 1.31 & 0.83 & $t(42)=1.34$ \\
\hline$(\mathrm{SD})$ & $(1.26)$ & $(0.99)$ & $p=.188$ \\
\hline STAI State overall change & 2.27 & 5.17 & $t(42)=1.02$ \\
\hline (SD) & $(9.41)$ & $(9.17)$ & $p=.188$ \\
\hline IPAQ Sedentary & 302.31 & 440.00 & $t(42)=3.02$ \\
\hline (mins/week; SD) & $(132.80)$ & $(169.39)$ & $p=.004$ \\
\hline
\end{tabular}


IPAQ Vigorous

106.15

166.67

$t(42)=1.711$

(mins/week; SD)

(120.38)

(107.54)

$p=.094$

Note. $\mathrm{SD}=$ standard deviation; $\mathrm{C} / \mathrm{NC}=$ Caucasian/Non-Caucasian; $\mathrm{Y} / \mathrm{N}=\mathrm{Yes} / \mathrm{No} ; \mathrm{F} / \mathrm{M} / \mathrm{L}=$

Follicular/Mid-luteal-Luteal; PHQ-9 = Patient Health Questionnaire; GAD-7 = Generalized

Anxiety Disorder questionnaire; STAI State = State-Trait Anxiety Inventory for Adults State; IPAQ

$=$ International Physical Activity Questionnaire. 


\section{Captions:}

Figure 1. Flowchart depicting participant attrition from the experiment and the reasons and various stages at which they were excluded.

Figure 2. Main effects of task (row 1), treatment (row 2) and their interaction (row 3 ) for HR (column 1) and HF HRV (column 2). Heart rate increases under stress (main effect of task), decreases during drug treatment (main effect of treatment) and decreases are greatest under stress, although this interaction effect was not significant. Heart rate variability decreases under stress (main effect of task), increases during drug treatment (main effect of treatment) and increases are greatest under rest (interaction effect). The lack of a significant effect for heart rate variability under stress reflects the difficulty of increasing parasympathetic function under stress.

Figure 3. Moderation of treatment effects by age during resting state (row 1) and stress (row 2) for HR (column 1) and HF HRV (column 2). While effects are significant for those aged 25 and over findings associated with a large effect size - they are not significant for those under 25 .

Figure 4. Individual participant data relating to attenuation of change in HR during stress with escitalopram. The attenuation of change in HR during stress with escitalopram was calculated using the following formula: (HR_rest_drug - HR_stress_drug) - (HR_rest_placebo HR_stress_placebo). A positive score on this measure represents greater attenuation of the stress with escitalopram (i.e. larger the score, the more attenuation). 


\section{Recruitment}

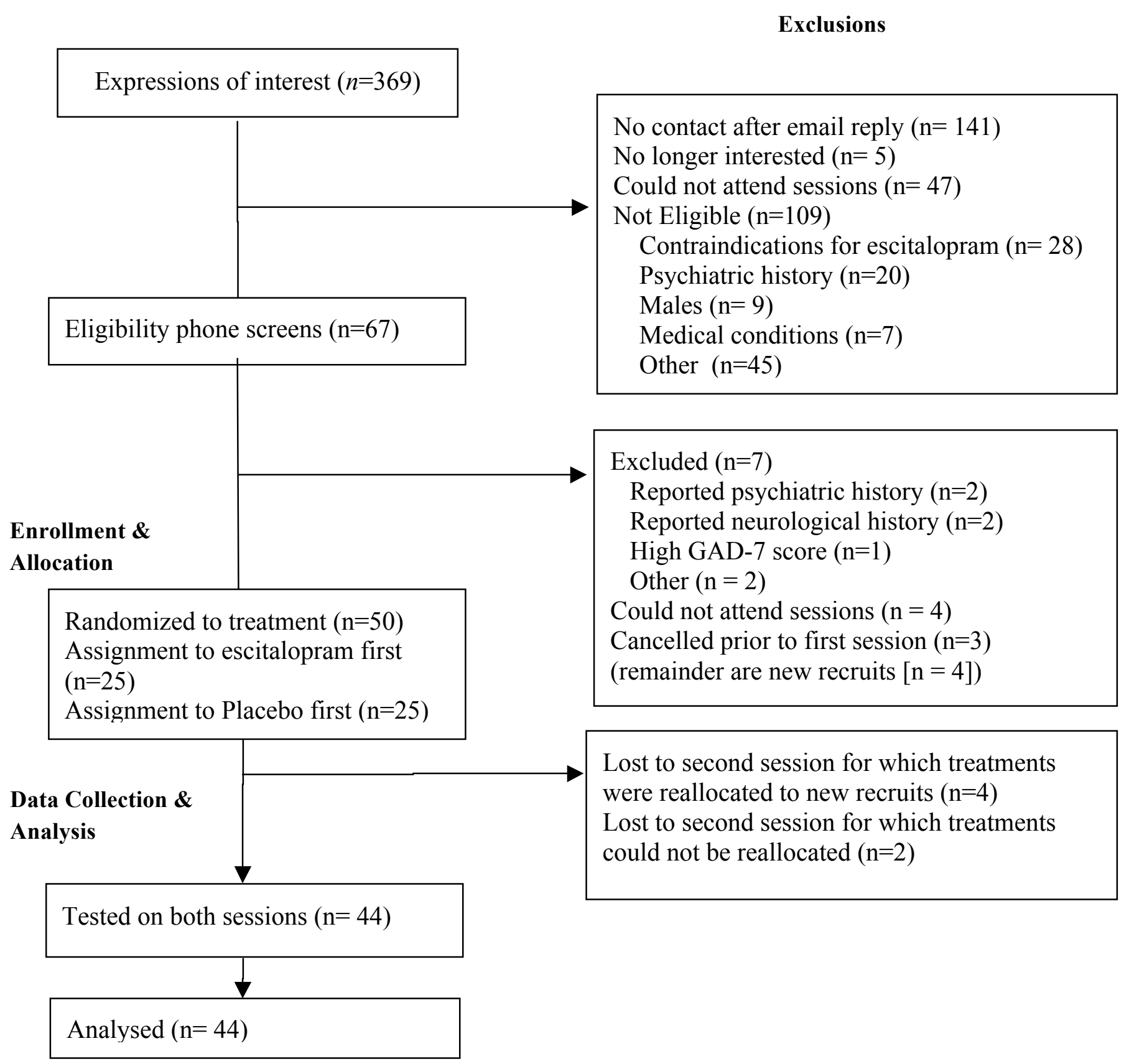

Figure 1. Participant attrition from the experiment and the reasons and various stages at which they were excluded (according to CONSORT guidelines). 
Heart Rate

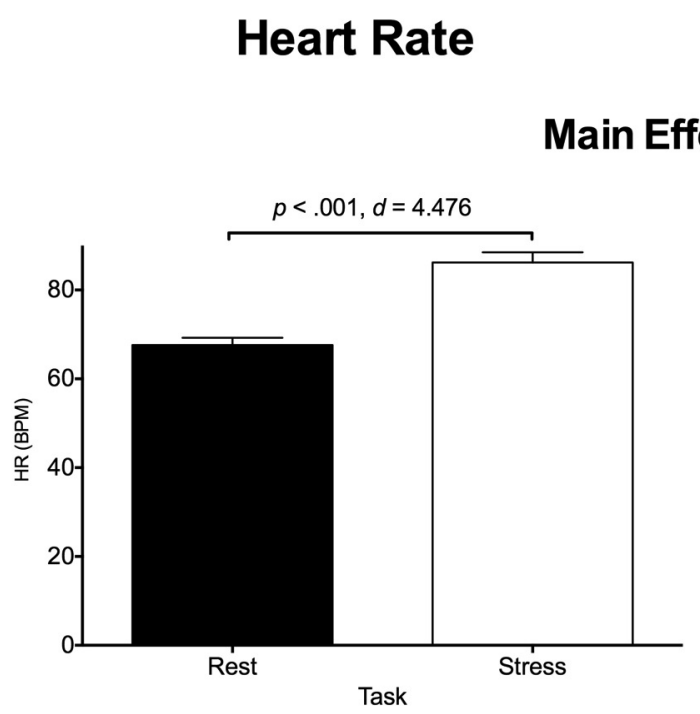

Heart Rate Variability

\section{ask}

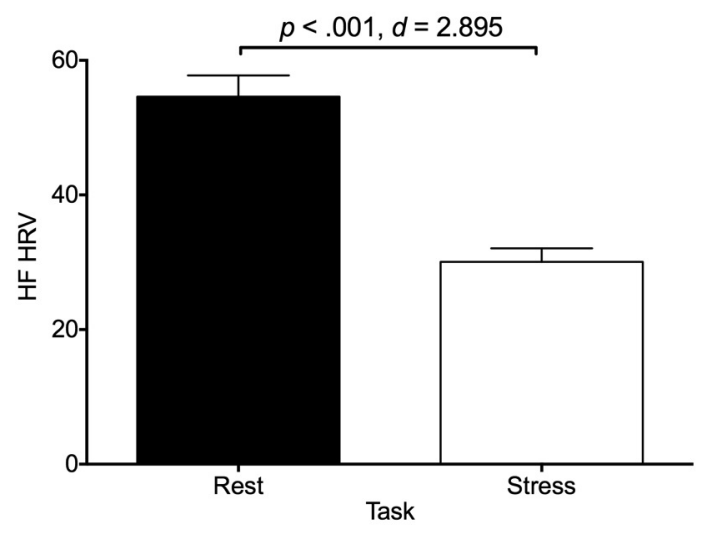

Main Effect of Treatment
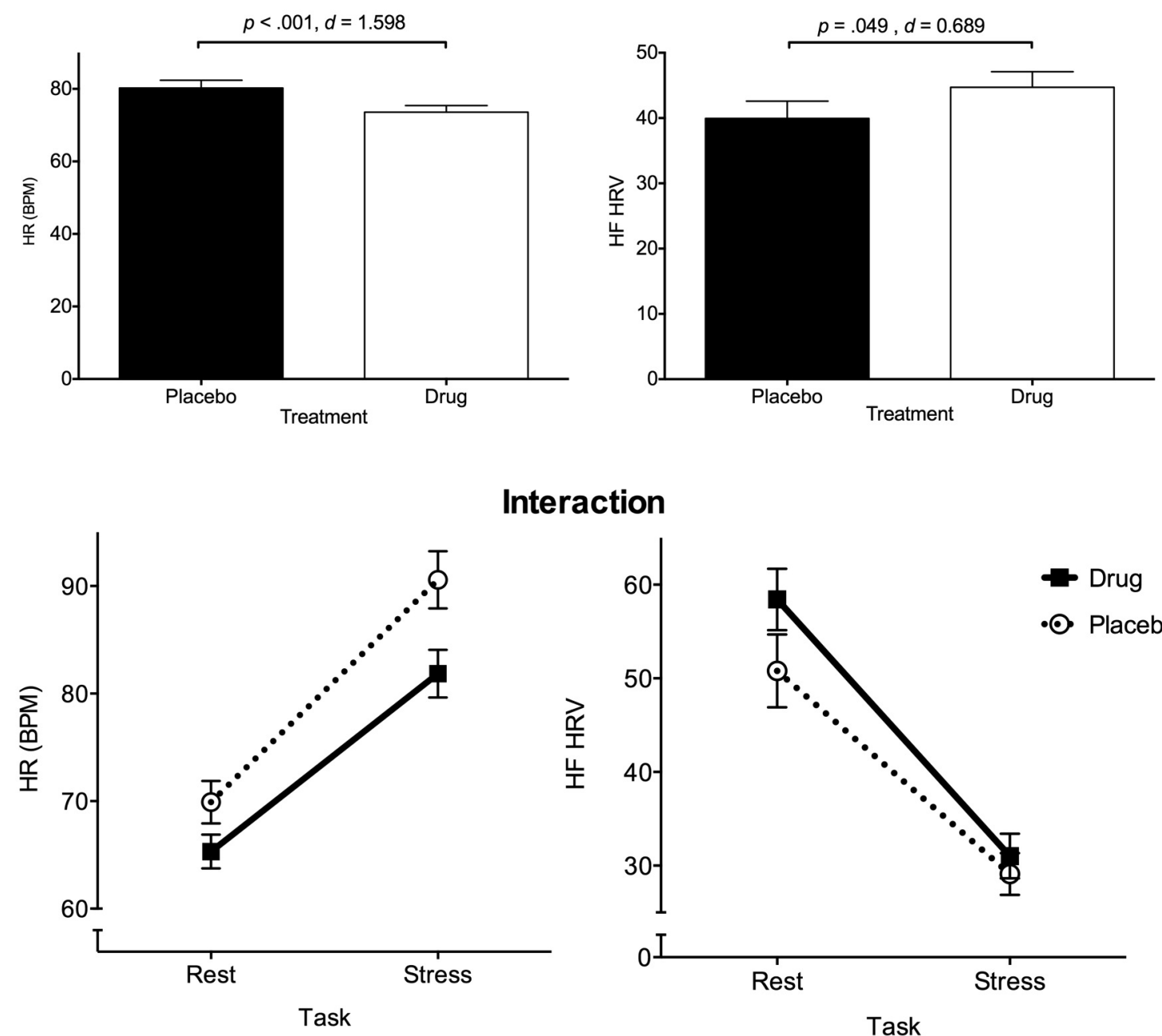

Interaction

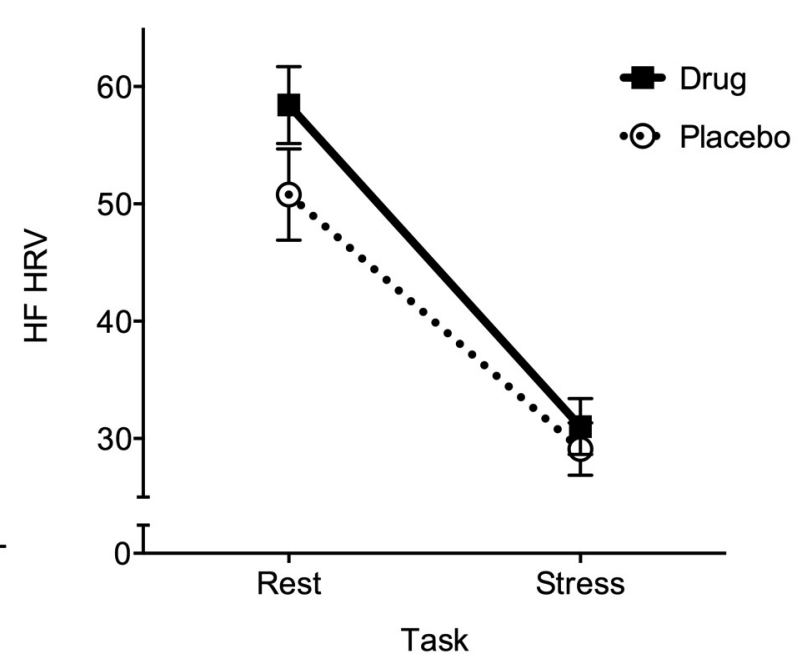

Fig 2 
Heart Rate

Heart Rate Variability

Rest

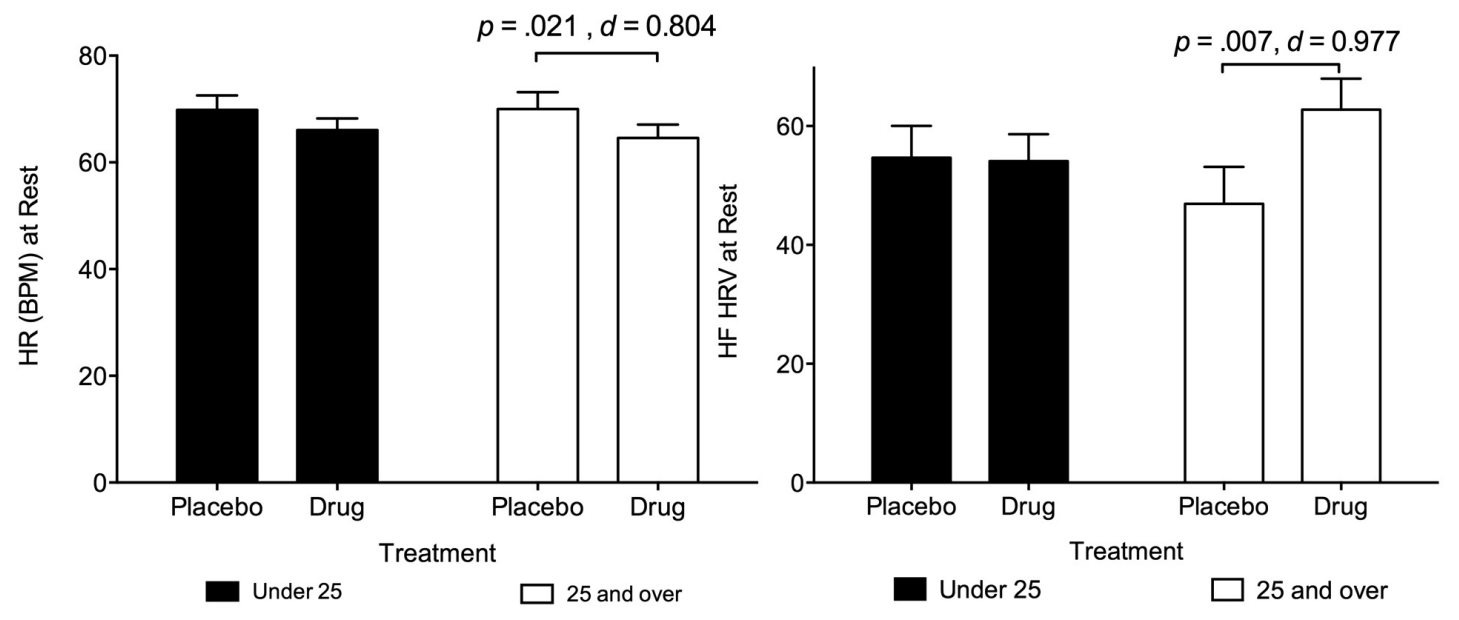

Stress
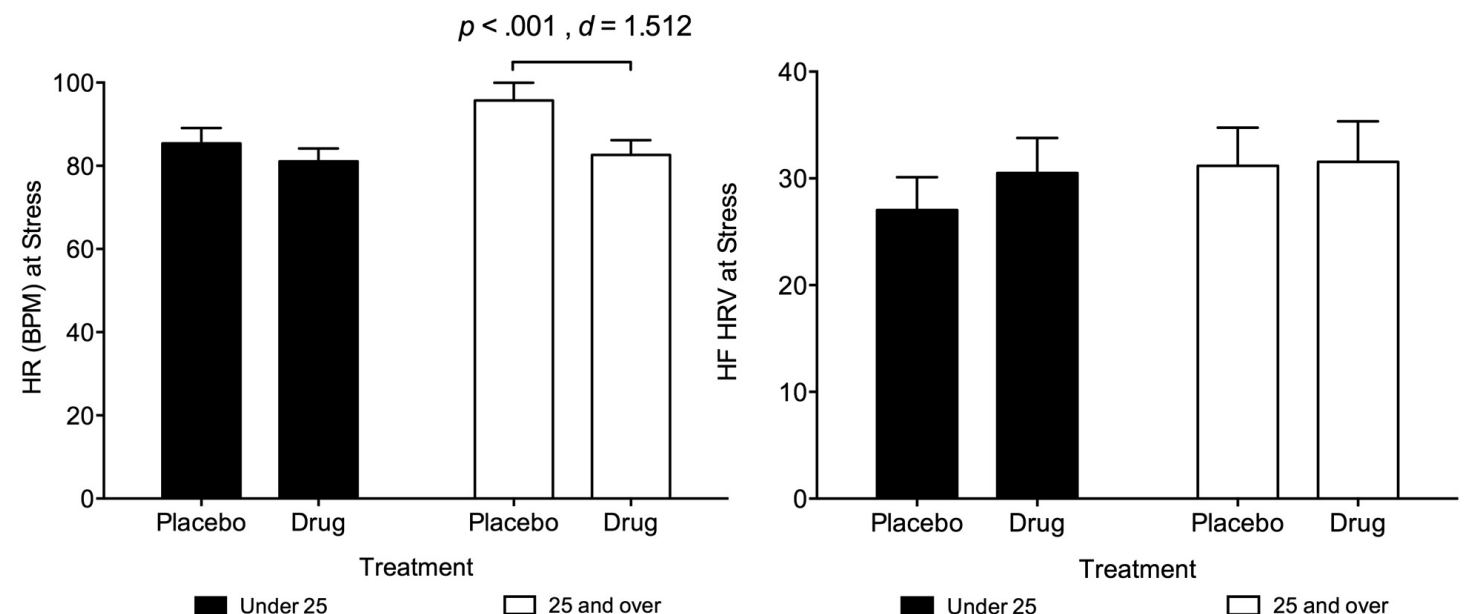

Fig 3 


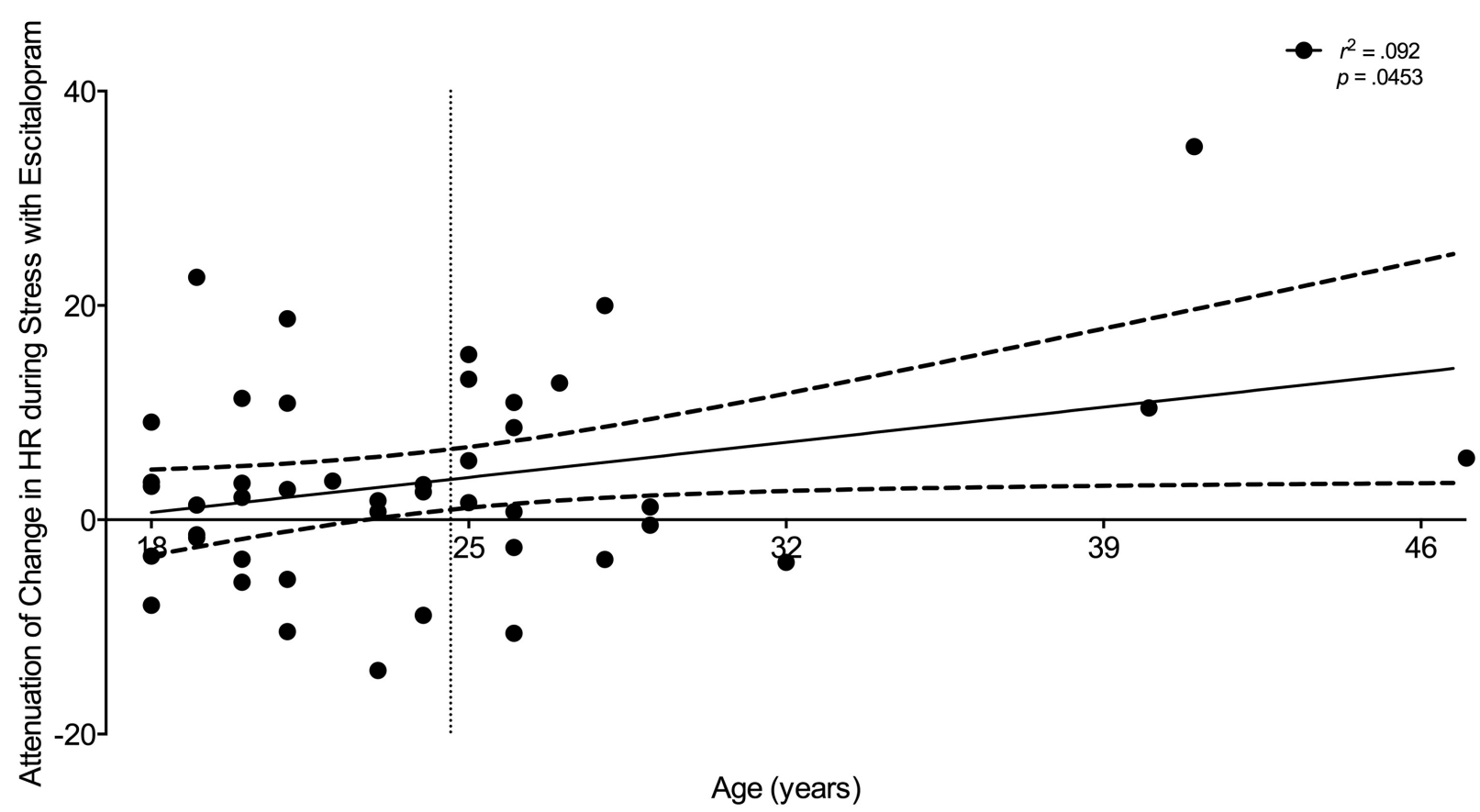

IntHR=(HR_rest_drug - HR_stress_drug) - (HR_rest_placebo - HR_stress_placebo).

Fig 4 


\section{References}

Ahn J-H, Patkar AA (2011) Escitalopram for the treatment of major depressive disorder in youth. Expert Opin Pharmacother 12:2235-2244. doi: 10.1517/14656566.2011.604632

Altemus M (2006) Sex differences in depression and anxiety disorders: Potential biological determinants. Hormones and Behavior 50:534-538. doi: 10.1016/j.yhbeh.2006.06.031

Anonymous (2012) Loxalate (Escitalopram oxalate) Product Information. 1-23.

Åberg MAI, Nyberg J, Torén K, et al. (2013) Cardiovascular fitness in early adulthood and future suicidal behaviour in men followed for up to 42 years. Psychol Med 1-10. doi: $10.1017 / \mathrm{S} 0033291713001207$

Barbui C, Esposito E, Cipriani A (2009) Selective serotonin reuptake inhibitors and risk of suicide: a systematic review of observational studies. CMAJ 180:291-297. doi: 10.1503/cmaj.081514

Benes FM, Turtle M, Khan Y, Farol P (1994) Myelination of a key relay zone in the hippocampal formation occurs in the human brain during childhood, adolescence, and adulthood. Arch Gen Psychiatry 51:477-484.

Booij L, Swenne CA, Brosschot JF, et al. (2006) Tryptophan depletion affects heart rate variability and impulsivity in remitted depressed patients with a history of suicidal ideation. Biol Psychiatry 60:507-514. doi: 10.1016/j.biopsych.2006.02.010

Carrillo M, Ricci LA, Coppersmith GA, Melloni RH Jr (2009) The effect of increased serotonergic neurotransmission on aggression: a critical meta-analytical review of preclinical studies. Psychopharmacology 205:349-368.

Casey BJ, Jones RM, Hare TA (2008) The adolescent brain. Ann N Y Acad Sci 1124:111-126. doi: 10.1196/annals. 1440.010

Cipriani A, Cipriani A, Santilli C, et al. (2009) Escitalopram versus other antidepressive agents for depression - Cochrane Database of Systematic Reviews - Cipriani - Wiley Online Library.

Cohen J (1992) Statistical power analysis. Current Directions in Psychological Science 1:98-101.

Cohen J (1988) Statistical Power Analysis for the Behavioral Sciencies. Lawrence Erlbaum Associates, Hillsdale, New Jersey

Cohen MJ, Branch WB, Willis WG, et al. (1992) Childhood. In: Puente AE, McCaffrey RJ (eds) Handbook of Neuropsychological Assessment: A Biopsychosocial Perspective. Plenum, New York, pp 49-79

Côté F, Fligny C, Fromes Y, et al. (2004) Recent advances in understanding serotonin regulation of cardiovascular function. Trends Mol Med 10:232-238. doi: 10.1016/j.molmed.2004.03.007

Crews F, He J, Hodge C (2007) Adolescent cortical development: a critical period of vulnerability for addiction. Pharmacol Biochem Behav 86:189-199. doi: 10.1016/j.pbb.2006.12.001

Davey CG, Yücel M, Allen NB (2008) The emergence of depression in adolescence: development of the prefrontal cortex and the representation of reward. Neurosci Biobehav Rev 32:1-19. doi: 
10.1016/j.neubiorev.2007.04.016

Depue RA, Spoont MR (1986) Conceptualizing a serotonin trait. A behavioral dimension of constraint. Ann N Y Acad Sci 487:47-62.

Fuster J (2008) Serotonin. In: The Prefrontal Cortex, 4 ed. Academic Press, London, pp 85-87

Gamelin FX, Berthoin S, Bosquet L (2006) Validity of the polar S810 heart rate monitor to measure $\mathrm{R}-\mathrm{R}$ intervals at rest. Medicine and science in sports and exercise 38:887-893. doi: 10.1249/01.mss.0000218135.79476.9c

Gibbons RD, Brown CH, Hur K, et al. (2012) Suicidal Thoughts and Behavior With Antidepressant Treatment: Reanalysis of the Randomized Placebo-Controlled Studies of Fluoxetine and Venlafaxine. Arch Gen Psychiatry. doi: 10.1001/archgenpsychiatry.2011.2048

Hammad TA, Laughren T, Racoosin J (2006a) Suicidality in pediatric patients treated with antidepressant drugs. Arch Gen Psychiatry 63:332-339. doi: 10.1001/archpsyc.63.3.332

Hammad TA, Laughren T, Racoosin J (2006b) Suicidality in pediatric patients treated with antidepressant drugs. Arch Gen Psychiatry 63:332-339. doi: 10.1001/archpsyc.63.3.332

Hanson CS, Outhred T, Brunoni AR, et al. (2013) The impact of escitalopram on vagally mediated cardiovascular function to stress and the moderating effects of vigorous physical activity: a randomized controlled treatment study in healthy participants. Frontiers in Physiology 4:259. doi: 10.3389/fphys.2013.00259

Harmer CJ, Goodwin GM, Cowen PJ (2009) Why do antidepressants take so long to work? A cognitive neuropsychological model of antidepressant drug action. The British Journal of Psychiatry 195:102-108. doi: 10.1192/bjp.bp.108.051193

Hogg S, Michan L, Jessa M (2006) Prediction of anti-panic properties of escitalopram in the dorsal periaqueductal grey model of panic anxiety. Neuropharmacology 51:141-145. doi: 10.1016/j.neuropharm.2006.03.009

Karanges E, Li KM, Motbey C, et al. (2011) Differential behavioural and neurochemical outcomes from chronic paroxetine treatment in adolescent and adult rats: a model of adverse antidepressant effects in human adolescents? Int J Neuropsychopharm 14:491-504. doi: $10.1017 / \mathrm{S} 146114571100006 \mathrm{X}$

Karanges E, McGregor IS (2011a) Antidepressants and adolescent brain development. Future Neurology 6:783-808. doi: 10.2217/fnl.11.51

Karanges E, McGregor IS (2011b) Antidepressants and adolescent brain development. Future Neurology 6:783-808. doi: 10.2217/fnl.11.51

Karanges EA, Kashem MA, Sarker R, et al. (2013) Hippocampal protein expression is differentially affected by chronic paroxetine treatment in adolescent and adult rats: a possible mechanism of "paradoxical" antidepressant responses in young persons. Front Pharmacol 4:86. doi: 10.3389/fphar.2013.00086

Kashdan TB, Rottenberg J (2010) Psychological flexibility as a fundamental aspect of health. Clin Psychol Rev 30:865-878. doi: 10.1016/j.cpr.2010.03.001 
Kasper S, Sacher J, Klein N, et al. (2009) Differences in the dynamics of serotonin reuptake transporter occupancy may explain superior clinical efficacy of escitalopram versus citalopram. International clinical psychopharmacology 24:119-125. doi: 10.1097/YIC.0b013e32832a8ec8

Kemp A, Gray M, Silberstein R, et al. (2004) Augmentation of serotonin enhances pleasant and suppresses unpleasant cortical electrophysiological responses to visual emotional stimuli in humans. NeuroImage 22:1084-1096. doi: 10.1016/j.neuroimage.2004.03.022

Kemp AH, Nathan PJ (2004) Acute augmentation of serotonin suppresses cardiovascular responses to emotional valence. Int J Neuropsychopharm 7:65-70. doi: 10.1017/S1461145703003894

Kemp AH, Quintana DS (2013) The Relationship Between Mental and Physical Health: Insights from the Study of Heart Rate Variability. International Journal of Psychophysiology 89:288296. doi: 10.1016/j.ijpsycho.2013.06.018

Khan A, Brodhead AE, Schwartz KA, et al. (2005) Sex Differences in Antidepressant Response in Recent Antidepressant Clinical Trials. Journal of Clinical Psychopharmacology 25:318-324. doi: 10.1097/01.jcp.0000168879.03169.ce

Kirschbaum C, Pirke KM, Hellhammer DH (1993) The "Trier Social Stress Test--"a tool for investigating psychobiological stress responses in a laboratory setting. Neuropsychobiology 28:76-81.

Kroenke K, Spitzer RL, Williams JB (2001) The PHQ-9: validity of a brief depression severity measure. J Gen Intern Med 16:606-613.

Lemogne C, Thomas F, Consoli SM, et al. (2011) Heart rate and completed suicide: evidence from the IPC cohort study. Psychosom Med 73:731-736. doi: 10.1097/PSY.0b013e3182365dc7

Li Z, Snieder H, Su S, et al. (2009) A longitudinal study in youth of heart rate variability at rest and in response to stress. Int J Psychophysiol 73:212-217. doi: 10.1016/j.ijpsycho.2009.03.002

Licht CMM, de Geus EJC, van Dyck R, Penninx BWJH (2010) Longitudinal evidence for unfavorable effects of antidepressants on heart rate variability. Biol Psychiatry 68:861-868. doi: 10.1016/j.biopsych.2010.06.032

Mann JJ (1998) The neurobiology of suicide. Nat Med 4:25-30.

Mann JJ (2003) Neurobiology of suicidal behaviour. Nat Rev Neurosci 4:819-828. doi: $10.1038 /$ nrn 1220

Mills EJ, Chan A-W, Wu P, et al. (2009) Design, analysis, and presentation of crossover trials. Trials 10:27. doi: 10.1186/1745-6215-10-27

Moll GH, Mehnert C, Wicker M, et al. (2000) Age-associated changes in the densities of presynaptic monoamine transporters in different regions of the rat brain from early juvenile life to late adulthood. Brain Res Dev Brain Res 119:251-257.

Murrin LC, Sanders JD, Bylund DB (2007) Comparison of the maturation of the adrenergic and serotonergic neurotransmitter systems in the brain: implications for differential drug effects on juveniles and adults. Biochemical Pharmacology 73:1225-1236. doi:

10.1016/j.bcp.2007.01.028 
Oldehinkel AJ, Verhulst FC, Ormel J (2008) Low heart rate: a marker of stress resilience. The TRAILS study. Biol Psychiatry 63:1141-1146. doi: 10.1016/j.biopsych.2007.12.006

Pohl R, Balon R, Jayaraman A, et al. (2003) Effect of fluoxetine, pemoline and placebo on heart period and QT variability in normal humans. Journal of psychosomatic research 55:247-251.

Puig MV, Gulledge AT (2011) Serotonin and Prefrontal Cortex Function: Neurons, Networks, and Circuits. Mol Neurobiol 44:449-464. doi: 10.1007/s12035-011-8214-0

Quintana DS, Heathers JAJ, Kemp AH (2012) On the validity of using the Polar RS800 heart rate monitor for heart rate variability research. Eur J Appl Physiol 112:4179-4180. doi: $10.1007 / \mathrm{s} 00421-012-2453-2$

Rao N (2007) The clinical pharmacokinetics of escitalopram. Clin Pharmacokinet 46:281-290.

Robbins TW, Crockett MJ (2009) Role of central serotonin in impulsivity and compulsivity: comparative studies in experimental animals and humans. In: Müller CP, Jacobs BL (eds) Handbook of the Behavioral Neurobiology of Serotonin. Academic Press, London, pp 415-427

Senn S (1994) The AB/BA crossover: past, present and future? Stat Methods Med Res

Senn S, D'Angelo G, Potvin D (2004) Carry-over in cross-over trials in bioequivalence: theoretical concerns and empirical evidence - Senn - 2004 - Pharmaceutical Statistics - Wiley Online Library. Pharmaceutical Statistics

Sogaard B (2005) The Pharmacokinetics of Escitalopram After Oral and Intravenous Administration of Single and Multiple Doses to Healthy Subjects. The Journal of Clinical Pharmacology 45:1400-1406. doi: 10.1177/0091270005280860

Sowell ER, Thompson PM, Holmes CJ, et al. (1999) In vivo evidence for post-adolescent brain maturation in frontal and striatal regions. Nat Neurosci 2:859-861. doi: 10.1038/13154

Spitzer RL, Kroenke K, Williams JBW, Löwe B (2006) A brief measure for assessing generalized anxiety disorder: the GAD-7. Arch Intern Med 166:1092-1097. doi:

10.1001/archinte.166.10.1092

Stone M, Laughren T, Jones ML, et al. (2009a) Risk of suicidality in clinical trials of antidepressants in adults: analysis of proprietary data submitted to US Food and Drug Administration. BMJ 339:b2880-b2880. doi: 10.1136/bmj.b2880

Stone M, Laughren T, Jones ML, et al. (2009b) Risk of suicidality in clinical trials of antidepressants in adults: analysis of proprietary data submitted to US Food and Drug Administration. BMJ 339:b2880-b2880. doi: 10.1136/bmj.b2880

Straneva-Meuse P (2004) Bupropion and paroxetine differentially influence cardiovascular and neuroendocrine responses to stress in depressed patients. J Affect Disord 79:51-61. doi: 10.1016/S0165-0327(02)00352-X

Thayer JF, Hansen AL, Saus-Rose E, Johnsen BH (2009) Heart rate variability, prefrontal neural function, and cognitive performance: the neurovisceral integration perspective on selfregulation, adaptation, and health. Ann Behav Med 37:141-153. doi: 10.1007/s12160-0099101-z 
Weippert M, Kumar M, Kreuzfeld S, et al. (2010) Comparison of three mobile devices for measuring R-R intervals and heart rate variability: Polar S810i, Suunto t6 and an ambulatory ECG system. Eur J Appl Physiol 109:779-786. doi: 10.1007/s00421-010-1415-9

Yakovlev PI, Lecours AR (1967) The myelogenetic cycles of regional maturation of the brain. In: Minskowski A (ed) Regional Development of the Brain in Early Life. Blackwell Scientific, Oxford, pp 3-70 